\title{
Prevalence, identification and antifungal susceptibility pattern of Candida species isolated from Iranian HIV patients with oropharyngeal candidiasis
}

Maryam Roudbary $^{1 *}$, Soleiman Khedri ${ }^{2}$, André L.S. Santos ${ }^{3}$,Ramtin Hadighi ${ }^{1}$, Mehraban Falahati ${ }^{1}$, Shirin Farahyar ${ }^{1}$, Majid khoshmirsafa ${ }^{4}$, Saeed Kalantari ${ }^{5}$

1Department of Medical Mycology and Parasitology, School of Medicine, Iran University of Medical Sciences, Tehran, Iran. 2Department of Medical Mycology and Parasitology, International Campus, Iran University of Medical Sciences, Tehran, Iran. 3Department of General Microbiology, Microbiology Institute, Federal University of Rio de Janeiro, Rio de Janeiro, Brazil. 4Department of Immunology, School of Medicine, Iran University of Medical Sciences, Tehran, Iran

5 Antimicrobial Resistance Research Center, Iran University of Medical Sciences, Tehran ,Iran

\section{Objectives:}

Candidiasis is an important opportunistic fungal infection in hospitalized patients as well as in immunocompromised individuals especially in cancer-treated patients, organ transplant recipients and HIV-infected persons. Oropharyngeal candidiasis (OPC) is a common mucocutaneous infection in more than $90 \%$ of the HIV-infected patients, particularly in the early and advanced stages of AIDS. In this study we identified the Candida species isolated from oral cavity of Iranian HIV/AIDS patients by conventional and molecular methods, as well as antifungal susceptibility test for Candida strains against five antifungal agents examined

\section{Methods:}

Herein, Specimens were obtained by sterile cotton swab from the tongue and buccal mucosa lesions from 150 HIV-positive patients admitted at the HIV Health Centers in Tehran,Iran. The samples cultured on Sabouraud-dextrose agar and identified by conventional , PCR amplification and Sequencing of ITS region. Minimum inhibitory concentration (MIC) of 102 Candida spp. was performed against itraconazole, fluconazole, voriconazole, caspofungin, and amphotericin B, using the broth microdilution assay according to the Clinical and Laboratory Standard Institute (CLSI; protocol M27-S3). Breakpoint for fluconazole, voriconazole, caspofungin provided by CLSI M27-S4 recommendation.

\section{Results}

Eighty nine patients (59.3\%) had positive culture for Candida and presented clinical signals of classical oral candidiasis. In this group, 102 morphologically distinct colonies were recovered and subsequently identified by polymerase chain reaction (PCR) and sequencing assay, presenting the following frequency: 54 C. albicans (52.9\%), 16 C. dubliniensis (15.7\%), 12 C. tropicalis (11.8\%), 9 C. glabrata (8.8\%), 7 C. kefyr (6.9\%) and 4 C. africana (3.9\%). Additionally, multiple Candida species were coisolated from $13.5 \%$ (12/89) patients. Regarding the antifungal susceptibility test, all Candida isolates were susceptible to amphotericin $\mathrm{B}$ and caspofungin, while some of them were resistant to fluconazole $(17.6 \% ; 16$ C. albicans, 1 C. dubliniensis and 1 C. glabrata), itraconazole (16.7\%; 15 C. albicans, 1 C. dubliniensis and 1 C. tropicalis) and voriconazole (5.9\%; 5 C. albicans and 1 C. tropicalis).

\section{Conclusion:}

Taken together, our finding displayed that HIV/AIDS patients are susceptible to oral candidiasis-related to host factors. Early and accurate detection of OPC could result in better outcome in infected patients. Even with the prophylaxis and treatment with antifungal agents, the increasing of resistance to common antifungal drugs in HIV-infected individuals has been noticed.finding reinforce the urgent necessity to address alternative therapeutic agents for treating oral candidiasis in HIVpositive patients due to the high incidence of azole-resistant $C$. albicans strains and the increase frequency of non-albicans Candida species.

Keywords: HIV;AIDS;Oropharyngeal candidiasis; Candida species; Antifungal susceptibility 